\title{
Sex differences in resting hemodynamics and arterial stiffness following 4 weeks of resistance versus aerobic exercise training in individuals with pre-hypertension to stage 1 hypertension
}

Scott R Collier ${ }^{1 *}$, Vincent Frechette ${ }^{2}$, Kathryn Sandberg ${ }^{4}$, Patrick Schafer ${ }^{1}$, Hong $\mathrm{Ji}^{4}$, Harold Smulyan ${ }^{2}$ and Bo Fernhall ${ }^{3}$

\begin{abstract}
Background: Hypertension (HTN) exhibits sexual dimorphism; the incidence for women surpasses men during the sixth decade of life, while the pharmacological treatments are less effective and produce more side-effects in women than in men. Aerobic exercise (AE) has been shown to prevent and treat HTN; however, resistance exercise (RE) is not recommended as a strategy to treat HTN. In this study, we investigated the potential sex differences of $A E$ versus RE in a cohort of unmedicated patients with hypertension.
\end{abstract}

Methods: In total, 40 moderately active, pre-hypertensive or stage 1 essential hypertensive male (M) and female (F) participants aged 40 to 60 years were randomly divided into four groups: M AE, M RE, F AE, and F. Each group exercised at moderate intensity, 3 days/week for 4 weeks. Hemodynamic, vascular and blood-flow data were collected before and after exercise training.

Results: Men showed a significant increase in central pulse wave velocity following RE while females showed no significant changes (12 \pm to $13.9 \pm$ vs. $9.2 \pm$ to $9.6 \pm \mathrm{m} / \mathrm{s}$, respectively). RE showed significantly greater increases in peak blood flow when compared to AE (F RE $15 \pm$ to $20 \pm$ vs. F AE $17.5 \pm$ to $19.5 \pm$, M RE $19 \pm$ to $24 \pm$ VS M AE 21 \pm to $25 \pm \mathrm{ml}^{*} 100 \mathrm{ml} \mathrm{F}^{*} \mathrm{~min}$, respectively). In addition, systolic and diastolic BP decreased greater for women following RE when compared to AE whereas men showed comparable decreases in BP following either exercise mode.

Conclusion: Moderate-intensity RE training may be a more favorable for women as a treatment option for hypertension because of greater decreases in diastolic BP and significant increases in flow-mediated dilation without concomitant increases in arterial stiffness, compared with their male counterparts.

\section{Background}

Hypertension exhibits sexual dimorphism; women have lower systolic blood pressure (SBP) levels than men from puberty through their mid-fifties, whereas the opposite is true after the sixth decade of life [1]. Hypertension is not only more prevalent in women than in men, but also more severe and less amenable to control, especially in older women [2].

\footnotetext{
* Correspondence: colliersr@appstate.edu

'Vascular Biology and Autonomic Studies Laboratory, Appalachian State

University, 111 Rivers Street, Boone, NC 28608, USA

Full list of author information is available at the end of the article
}

Moderate-intensity aerobic exercise (AE) is recommended as a non-pharmacological therapy for the management of raised BP because it reduces both SBP and diastolic blood pressure (DBP), and improves arterial function in pre- to stage 1 essential hypertension in both sexes [3]. Our laboratory previously showed that resistance exercise (RE) also reduced $B P$ but to a slightly lesser extent than $\mathrm{AE}$ in a study with a predominantly male population [3]. RE training may be of greater importance to women because this mode of training increases bone-mineral density, which has been shown to protect against osteoporosis [4,5], and serves to

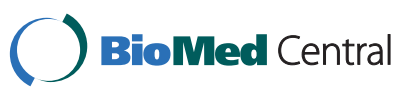

(c) 2011 Collier et al; licensee BioMed Central Ltd. This is an Open Access article distributed under the terms of the Creative Commons Attribution License (http://creativecommons.org/licenses/by/2.0), which permits unrestricted use, distribution, and reproduction in any medium, provided the original work is properly cited. 
increase muscle strength and endurance, which can prevent or delay the risk of disability in aging women $[5,6]$. However, RE training may also increase arterial stiffness, which would be a deleterious effect. The only available study in unmedicated patients with hypertension showed that arterial stiffness increased after RE [3]; however, this study was conducted in a primarily male population.

Recently, it has been shown that older men have increased blood flow after AE training, and that endothelial function is preserved in trained subjects after acute RE, which may decrease resting BP even in the presence of increases in arterial stiffness $[7,8]$. This is an important point to consider for individuals presenting with hypertension because many have increased arterial stiffness resulting from their condition. Although many studies have been conducted in normotensive and exercise-trained populations, relatively little is known about the effect of RE training on BP and arterial function in women with hypertension. Therefore, the purpose of this study was to compare the effects of 4 weeks of $\mathrm{AE}$ versus $\mathrm{RE}$ in both women and men with hypertension who were not on anti-hypertensive medications. We hypothesize that women would show greater increases in blood flow and greater decreases in BP without concomitant increases in arterial stiffness.

\section{Methods}

The protocol was approved by the University institutional review board, and all subjects gave written informed consent before participation.

\section{Subjects}

In total, 40 moderately active subjects $(20$ men, 20 women) aged 40 to 60 years, who were pre-hypertensive (120 to 139 systolic and/or 80 to $89 \mathrm{mmHg}$ diastolic BP) or or had stage 1 essential hypertension(140 to 149 systolic and/or 90 to $99 \mathrm{mmHg}$ diastolic) were recruited by local physicians and screened for medical and health histories [9]. The female group consisted of postmenopausal women only, to avoid the influence of hormonal changes, and they were required to have a history of more than 12 months of amenorrhea and no use of hormone-replacement therapy. Subject baseline characteristics are shown in Table 1.

On average, men are diagnosed with hypertension at an earlier age than women, therefore age-matched men might have undergone structural and functional changes that would confound our results. Thus, we chose to consider how long a subject had lived with the disease. Each subject consented to their blood-pressure history being provided by their physician, including the length of time the subject had been at or above pre-hypertension levels. All subjects enrolled in the study had been recently diagnosed (within 1 year) as having pre-
Table 1 Descriptive characteristics ${ }^{\mathrm{a}}$

\begin{tabular}{lllll}
\hline & RE M & AE M & RE F & AE F \\
\hline Age, years & $44 \pm 1.5$ & $46 \pm 1.5$ & $52 \pm 1.5^{*}$ & $54 \pm 1.5^{*}$ \\
Stature cm & $177 \pm 1^{*}$ & $179 \pm 1^{*}$ & $160 \pm 1$ & $162 \pm 1$ \\
Mass, kg & $101 \pm 5^{*}$ & $97 \pm 5^{*}$ & $72 \pm 5$ & $78 \pm 5$ \\
Body fat, \% & $31 \pm 2$ & $29 \pm 2$ & $40 \pm 2^{*}$ & $37 \pm 2^{*}$ \\
SBP, mmHg & $138 \pm 3.2$ & $136 \pm 4.2$ & $148 \pm 6^{*}$ & $147 \pm 4.5^{*}$ \\
DBP, $\mathrm{mmHg}$ & $78 \pm 2$ & $80 \pm 2$ & $78 \pm 3$ & $81 \pm 2$ \\
MAP, mmHg & $96 \pm 2$ & $99 \pm 2$ & $101 \pm 2$ & $103 \pm 2$ \\
RHR, beats/min & & & & \\
\multicolumn{1}{c}{ Before exercise } & $71 \pm 3$ & $75 \pm 3$ & $67 \pm 5$ & $69 \pm 4$ \\
$\quad$ After exercise & $73 \pm 3$ & $68 \pm 3$ & $68 \pm 5$ & $66 \pm 4$ \\
\hline
\end{tabular}

Abbreviations: $A E$, aerobic exercise; $D B P$, diastolic blood pressure; $F$, female; $R E$, resistance exercise, $M$, male; $M A P$, mean arterial pressure; RHR, resting heart rate; SBP systolic blood pressure.

All data are expressed as mean $\pm \mathrm{SE}$;

Asterisk $(*)$ denotes significance $(P \leq 0.05)$.

hypertension or stage 1 essential hypertension. Consequently, women were significantly older than men (8 years).

Using the medical histories obtained from physician records, it was confirmed that no subjects had a history of diabetes, kidney disease, atherosclerosis or hypercholesterolemia. None of the subjects was taking any medication, including antihypertensives or aspirin, and all were non-smokers.

\section{Study procedure}

Subjects reported to the laboratory at the same time of day, 3 hours after a meal, for each of the three measurement visits. Subjects were asked to refrain from caffeine and exercise before each visit. The first visit consisted of group randomization, and the taking of a health history and physical activity questionnaire, followed by tests of body composition, peak aerobic capacity $\left(\mathrm{VO}_{2 \text { peak }}\right)$ or 10 repetition maximum (10 RM) test, and measurement familiarization.

During the second visit, after 15 minutes of supine rest in a dimly lit, quiet room, pulse wave velocity (PWV) and $\mathrm{BP}$, followed by blood flow and reactive hyperemia were measured while the subject rested quietly. During the following 4 weeks, all subjects reported to a training facility, and each exercise session was supervised by an exercise physiologist. All subjects reported back to the laboratory for their post-training measurements at between 24 and 48 hours after completion of the last exercise session. All measurements were repeated at the same time of day in the postprandial state ( $>3$ hours) and in the same order as previous measurements.

\section{Anthropometrics}

Whole-body plethysmography (Bod Pod, Life Measurement Inc., Concord, CA, USA) was used to assess body 
composition [10], and body weight was measured using the Bod Pod scale to the nearest half-kilogram. Height was measured using a stadiometer to the nearest 0.5 $\mathrm{cm}$, and body mass index was calculated as: weight $(\mathrm{kg})$ divided by height $\left(\mathrm{m}^{2}\right)$.

\section{Maximal exercise testing}

Subjects randomized to the AE arm of the study completed a customized $\mathrm{VO}_{2 \text { peak }}$ protocol on a treadmill. Briefly, subjects started walking at 3 miles/hour for 2 minutes. Adjustments to the speed were made until the subject achieved a stable pace, at which point the grade was increased by $2 \%$ every 3 minutes until volitional fatigue was reached. Expired gases were analyzed using a breath-by-breath metabolic cart (Quark $b^{2}$; Cosmed, Rome, Italy). Rating of perceived exertion (RPE) and heart rate (HR) (Polar Electro Inc., Woodbury, NY, USA) were acquired once per stage. Maximal effort was achieved when subjects met three of the following criteria: 1) a final RPE score of $\geq 17$ on the Borg scale (rankings from 6 to 20); 2) a respiratory equivalent ratio (RER) of $>1.15$; 3 ) no change in HR after a change in workload; and/or 4) a plateau in oxygen uptake with an increase in workload $(<150 \mathrm{ml})$. The AE group underwent maximal $\mathrm{VO}_{2 \text { peak }}$ testing both before and after training.

Subjects randomized to the RE arm completed a 10 RM test. After a brief warm-up, an estimated load was given for each exercise (leg press, lateral pulldown, leg extension, chest press, leg curl, shoulder press, bicep curl, tricep press, and abdominal crunch), and each subject completed no fewer than 8 and no more than 15 repetitions. If the subject achieved fewer or more than 10 repetitions, prediction tables were used to add or reduce weight until the preferred load was attained. The subjects were again measured at the conclusion of their $\mathrm{RE}$ program to evaluate the effectiveness of the training protocol.

\section{Exercise training}

The AE training consisted of 30 minutes of treadmill exercise at $65 \%$ of the subject's previously determined $\mathrm{VO}_{\text {2peak }}, 3$ days per week for 4 weeks. The $10 \mathrm{RM}$ test provided the basis for individual load resistance for the dynamic RE sessions. The REs consisted of leg press, chest press, leg extension, lateral pulldown, leg curls, shoulder press, bicep curl, tricep press and abdominal crunch, all performed on the same equipment (Life Fitness, Schiller Park, IL, USA). Each subject completed three sets of ten repetitions at $65 \%$ of their 10 RM, 3 days/week for 4 weeks. Each RE session took approximately 45 to 50 minutes to complete. Subjects were asked to refrain from any exercise outside of their $\mathrm{AE}$ or RE sessions.

\section{Central and peripheral arterial stiffness}

PWV measurements were obtained with two MD6 bidirectional transcutaneous Doppler probes (Hokanson, Bellevue, WA, USA) in accordance with the guidelines of the Clinical Application of Arterial Stiffness Task Force 3 [11]. Each subject was monitored by electrocardiography (modified CM5), and HR data were gated in phase with the PWV measurements and used as timing markers for PWV identification. Central PWV measurements were obtained from the left common carotid artery to the left femoral artery. Distances from the carotid site to the mid-point of the manubrium sterni were subtracted from the carotid-to-femoral artery distance. Peripheral PWV measurements were obtained from the left femoral artery to the ipsilateral superior dorsalis pedis artery. The distance between each PWV location was obtained with a tape measure, and recorded to the nearest millimeter.

Data were collected in real time by aligning the Doppler waveforms and the electrocardiography tracings on a computer screen (MP100, BioPac Systems, Santa Barbara, CA, USA). All data were stored until analyzed at a later time. PWV was measured from the foot-to-foot flow wave velocity, whereas the foot of the sound wave was identified as the point of systolic upstroke. A minimum of 10 pulse contours were recorded and analyzed as the distances between points, and the time delay between proximal and distal foot waveforms was calculated as the distance (D) divided by the change in time $(\mathrm{m} / \mathrm{s})$. In our laboratory, one blinded technician analyzed all of the data, and the intra-class correlation coefficient for PWV, calculated using both central and peripheral sites on two separate days, was 0.98 .

\section{Hemodynamic monitoring}

In addition to physician visits, BP was measured twice (before and after training) through standard manual methods after the subject had rested quietly for 15 minutes in the seated position. During supine rest, BP was taken before and after the intervention with an automated auscultatory instrument (SunTech Medical Instruments, Raleight, NC, USA) method. All BP measurements were in accordance with American Heart Association standards. HR was taken from the calculation of successive $R-R$ intervals from the three-lead electrocardiograph.

\section{Blood flow and reactive hyperemia}

Forearm blood flow (FBF) and forearm reactive hyperemia (FRH) were measured using a mercury-in-silastic strain-gauge plethysmograph (EC-6, DE Hokanson, Inc., Issaquah, WA, USA) as described previously [12]. All data were transmitted to a computer, and analyzed 
(Non-Invasive Vascular Program 3 Software Package, version 5.27b; DE Hokanson). Six plethysmography measurements were averaged for baseline blood-flow values before and after exercise. The intra-assay coefficient of variation was $5.2 \%$. Following baseline flow measurements, a second occlusion cuff was placed over the first upper-arm cuff and inflated for 5 minutes at 250 $\mathrm{mmHg}$. Again, 1 minute before deflation of the upperarm cuff, a wrist cuff was inflated to $50 \mathrm{mmHg}$ above $\mathrm{SPB}$, which remained inflated until the end of the measurement. After 5 minutes of occlusion, the upper-arm cuff was rapidly deflated to induce reactive hyperemia. Following cuff release, 3 minutes of blood-flow measurements were recorded as described above. FBF was expressed as milliliter/min/100 $\mathrm{ml}$ of tissue. To avoid confounding other vascular measurements, $\mathrm{RH}$ was conducted after all other measurements were complete.

\section{Treatment of the data}

Vasodilatory capacity was calculated from the bloodflow data as the area under the curve (AUC) above baseline values using GraphPad Prism software (version 3.02;) and the trapezoidal rule on the basis of actual datum points. A one-way analysis of variance (ANOVA) with repeated measures (exercise mode (AE versus $\mathrm{RE}$ ) by time (before versus after training) by sex (male versus female)) was used with SPSS software (version 17; SPSS Inc. Chicago, IL, USA) on all dependent variables. If a significant interaction was detected, an appropriate post hoc test was conducted. The a priori significance was set at $\alpha<0.05$, and all data are reported as means \pm SEM.

The sample size of 40 subjects for the present study was based on previous data from our laboratory gathered under similar conditions. For these calculations, the STATA statistical software package (STATA, College Station, TX, USA) was used to determine the number of subjects to give us adequate statistical power at $P$ $<0.05$.

\section{Results}

Women had a significantly greater percentage of body fat and higher SBP than their male counterparts, while men had significantly greater stature and mass (RE 29 $\mathrm{kg}, \mathrm{AE} 19 \mathrm{~kg}$ ) than women.

There was a significant time $\times$ sex $\times$ mode interaction for central PWV $P=0.001$ ). Men had a significant increase in central PWV after RE, whereas women had no change in central PWV over time (Figure 1). Further, there was a significant decrease in PWV in both $\mathrm{AE}$ groups $(P=0.03)$. There were no significant sex differences in peripheral PWV (M AE $12.8 \pm 1.9$ versus 12.7 $\pm 1.9 \mathrm{RE} 13.2 \pm 1.8$ versus $13.9 \pm 1.8$, F AE $10.3 \pm 1.6$ versus $10.3 \pm 1.6$ and $F R E 9.4 \pm 1.5$ versus $9.6 \pm 1.5 \mathrm{~m} /$

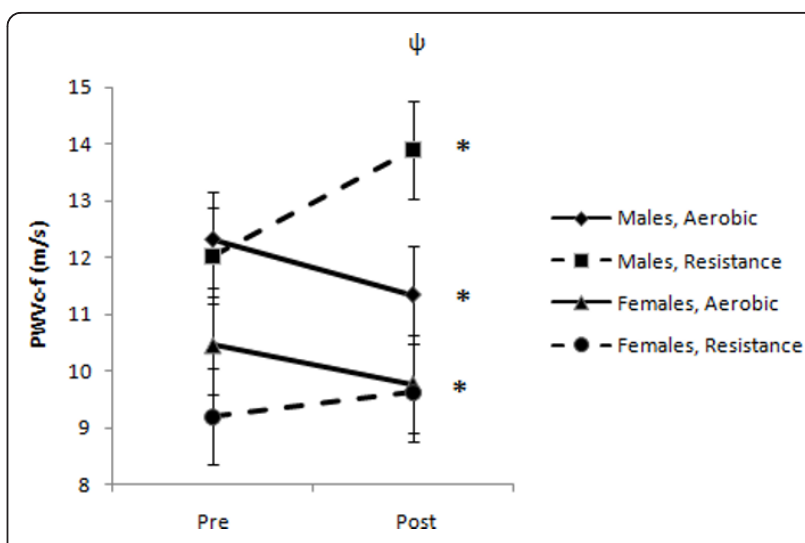

Figure 1 Changes in central pulse wave velocity (PWV) between men and women. There was a significant time $x$ sex by mode interaction for central PWV $(\psi, P=0.001)$. Men had a significant increase in central PWV after resistance exercise (RE), whereas PWV in women did not change over time. Further, there was a significant decrease in PWV in both aerobic exercise (AE) groups $(P=0.03)$

$\mathrm{s}$, before and after training respectively) after $\mathrm{RE}$ or $\mathrm{AE}$ training.

There was a significant timex mode $\times$ sex interaction $(\psi, P=0.01)$ as there was a greater increase in FBF after $\mathrm{RE}$ than after $\mathrm{AE}$, and women had a significantly greater increase than men after $\operatorname{RE}(P=0.04$ Figure $2 \mathrm{a})$. There was a significant increase in peak blood flow after both modes of exercise, but RE condition resulted in significantly higher peak blood flow ( $P=0.04$; Figure $2 \mathrm{~b}$ ). There was a significant increase in AUC of blood flow after training, and RE increased blood flow significantly more than $\mathrm{AE}$ in both cohorts $(\mathrm{M} \mathrm{AE} 94.8 \pm 10$ versus $128.7 \pm 12$, M RE 89.2 versus $139.4 \pm 12$, F AE 78.3 versus $97.3 \pm 9$ and F RE $79.4 \pm 10$ versus $110.6 \pm 12 \mathrm{ml} /$ $100 \mathrm{ml}^{\prime} \mathrm{min}$, before versus after training, respectively).

RE induced significant decreases in resting SBP $(P=$ $0.045)$ but not DBP for men, and there was a group $\times$ mode $\times$ time interaction for DBP in women $(\psi, P=$ 0.02; Figure 3a). Whereas AE produced significant training effects in both cohorts $(P=0.048)$, there were no significant group $\times$ mode differences or any significant decrease in DBP after RE training (Figure 3b).

\section{Discussion}

The results from this study suggest that $\mathrm{AE}$ has different effects on the cardiovascular system of men and women. The majority of studies assessing the effects of exercise training on BP and arterial function have been conducted in single-sex or mixed-sex populations, but without direct comparisons between the sexes [13-17]. To our knowledge, the present study is the first to directly compare differences between the sexes in the effects of exercise mode on resting hemodynamic parameters and 


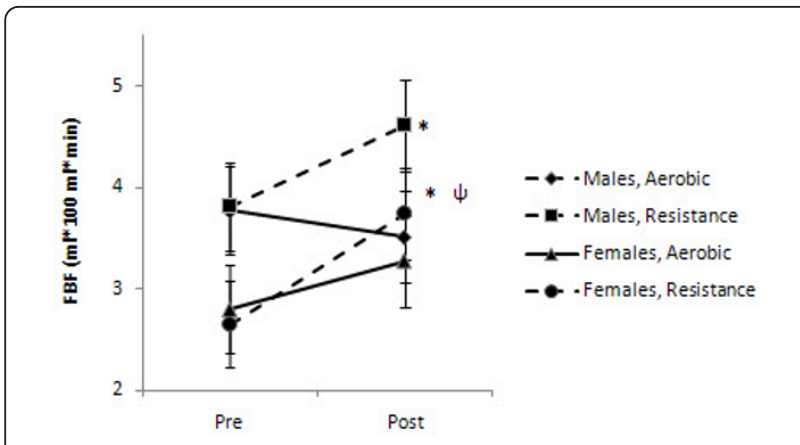

Figure 2b Peak forearm blood flow changes

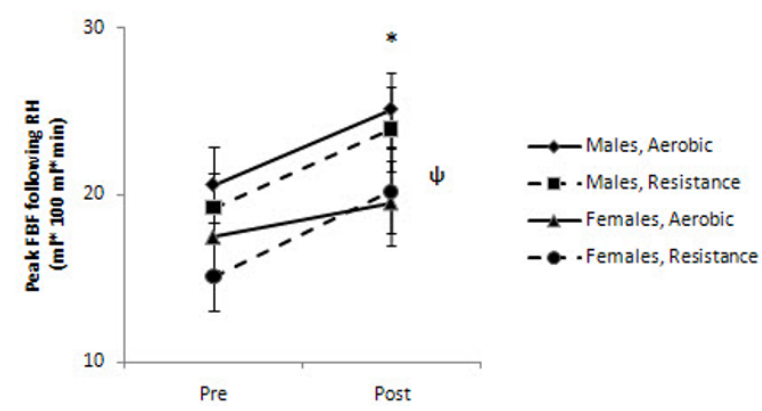

Figure 2 Changes in basal and peak forearm blood flow (FBF). (A) For basal FBF, there was a significant time $\times$ mode $\times$ sex interaction $(\psi, P=0.01)$ as resistance exercise (RE) produced greater increases in basal FBF compares with aerobic exercise (AE), and women had a significantly greater increase after RE than men $(P=$ 0.042). (B) For peak FBF, there was a significant increase in peak blood flow after both exercise modes, and RE induced significantly higher peak blood flow $(P=0.04)$.

variables of arterial stiffness in an unmedicated hypertensive cohort. The major novel finding of our study was that RE resulted in increases in central arterial stiffness in men, but not in women (Figure 1a,b). Conversely, AE produced a decrease in central arterial stiffness but no change in peripheral arterial stiffness for either sex. Another novel finding was that compared with $\mathrm{AE}$, $\mathrm{RE}$ resulted in a greater increase in basal and peak FBF in both men and women (Figure 2a,b).

Several studies support our present findings on arterial stiffness. Miyachi et al. [18] reported a 19\% increase in central arterial stiffness in a group of men who underwent a 4-month RE protocol [18]. Similarly, Bertovic et al. [19] found that men who used RE training regularly (consistently for a period of $>12$ months) had stiffer arteries than did their sedentary counterparts. One researcher reported RE with the upper body has been shown to increase arterial stiffness in pre-menopausal women [20], however, multiple investigations have shown RE to elicit no change in arterial stiffness in middle-aged women $[21,22]$. Not all studies have shown RE

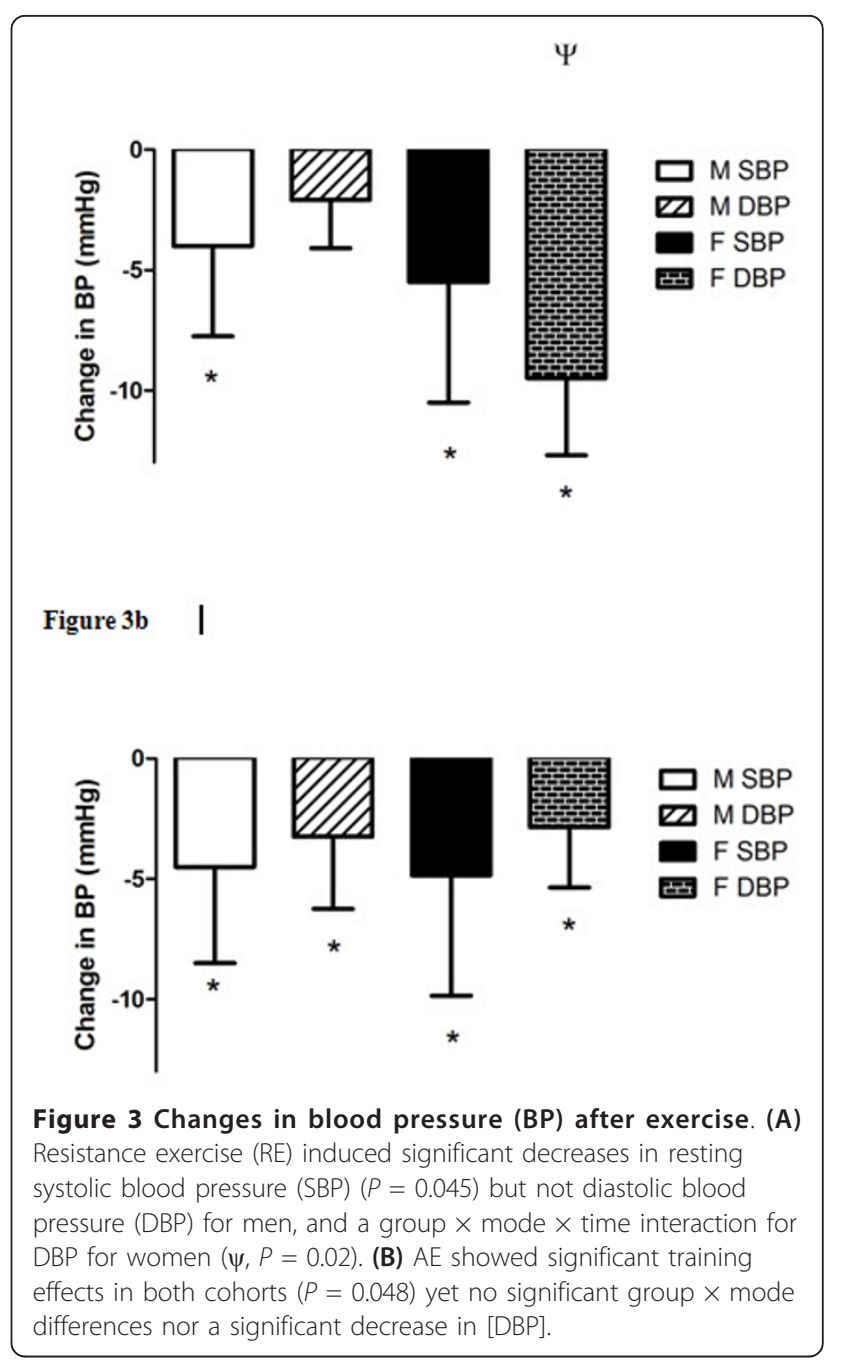

to yield increases in arterial stiffness in men and no change or a decrease in stiffness in women [21,23,24]. Cortez-Cooper et al. [25] found that high-intensity RE elicited an increase in arterial stiffness in women, whereas Rakobowchuk et al. [24] observed no change in arterial stiffness with RE in healthy men. However, none of these previous studies evaluated the effect of RE in an untreated hypertensive cohort.

Conversely, AE elicits favorable effects on the vasculature in both men and women. Moderate-intensity AE has been shown to increase arterial stiffness in older men [26] and in postmenopausal women [27]. Arterial stiffness increases with aging in sedentary individuals, yet this increase is attenuated with regular AE [28]. However, there is some evidence of a threshold effect, at which exercise becomes less effective in combating agerelated increases in arterial stiffness. Ferrier and colleagues [29] performed a study examining the effects of moderate-intensity exercise on arterial stiffness in an older mixed-sex population (mean age 64.7 years) with 
isolated systolic hypertension, and found that 8 weeks of moderate-intensity cycling did not stimulate the positive changes in arterial stiffness seen with exercise in any of the aforementioned studies. This suggests that the deleterious changes occurring in the vasculature with aging may become irreversible if proper interventions are not introduced and such processes are allowed to progress over time. Interestingly, women seem to have greater age-related increases in ventricular/vascular stiffness than men, even in the absence of cardiovascular disease [30]. This age-dependent increase in arterial stiffness with aging in women is believed to be a contributor to the accelerated increase in SBP that is prevalent in older women as they age. Thus, it is important to understand the effect of potential non-pharmacological therapies, such as exercise training, on both BP and arterial stiffness. It is important to note that many investigators have shown increases in central arterial stiffness after RE, but no changes in peripheral stiffness. This is important because the arterial baroreflex found in the aortic arch may be susceptible to desensitization if central arterial stiffness increases. This would lead to chronically higher sustained pressure and deleterious effects on the systemic arterial circulation. Importantly, despite discrepancies between men and women in exercise-induced arterial stiffness, both modes of exercise we used elicited beneficial changes in BP, some of which were similar to those seen in our previous study [3]. AE elicited a decrease of $4 \mathrm{mmHg} \mathrm{SBP}$ and $3 \mathrm{mmHg}$ DBP in men and decreases of 5 and $3 \mathrm{mmHg}$ in SBP and $\mathrm{DBP}$, respectively in women (Figure $3 \mathrm{a}, \mathrm{b}$ ). These changes mirror those seen in a meta-analysis by Fagard on the effects of $\mathrm{AE}$ on $\mathrm{BP}$ [31]; RE yielded decreases in SBP and DBP of 4 and $2 \mathrm{mmHg}$, respectively. These changes were similar to those seen in the meta-analysis by Kelley and Kelley [32]. Women also exhibited decreases in SBP and DBP with RE, but had a much greater decrease in DBP compared with men: -10 versus $-2 \mathrm{mmHg}$. This could be explained by the difference between the sexes in arterial stiffness after RE, as women did not exhibit an increase in arterial stiffness with RE. In addition, the lower muscle mass in women may derive less retrograde shear stress from RE compared with men, which may augment greater functional changes because of the attenuation of the blunted signaling on the endothelium [33,34].

The decrease in mean arterial pressure seen in men despite the increase in arterial stiffness suggests that the increase in flow, potentially caused by augmented vasodilation, is a protective compensatory mechanism. However, the flow increases with RE were identical in men and women. Furthermore, in both sexes, RE induced a greater increase than $\mathrm{AE}$ in basal FBF. It is currently known that functional changes resulting in increases in vascular conductance are endothelium-dependent, and are modulated by shear stresses on the vascular wall, augmenting the expression of endothelial nitric oxide synthase and extracellular superoxide dismutase, which act synergistically to increase the bioavailability of nitric oxide (NO) [35-37]. It is possible that the high force contractions produced by the skeletal muscle in RE may yield greater shear stresses on the vasculature, resulting in an increase in NO bioavailability at rest. Interestingly, the changes in basal FBF with RE did not translate to a concomitant increase in peak vasodilatory capacity in men, as peak FBF after RH was nearly identical with both modes of exercise. However, the changes in female $\mathrm{RH}$ were greater after RE than AE, which may result in greater blood flow and greater decreases in BP compared with their male counterparts.

\section{Conclusion}

This investigation shows that both $\mathrm{AE}$ and RE elicit beneficial changes in resting $\mathrm{BP}$ in both men and women. However, RE produced increases in central arterial stiffness in men with hypertension, whereas no such changes were shown in the women. These changes in stiffness were offset by a concurrent enhancement in vascular conductance, as RE elicited greater increases in blood flow compared with $\mathrm{AE}$ in both sexes. Therefore, $\mathrm{RE}$ can be a beneficial addition to the treatment plan in both men and women with hypertension. With exercise being a beneficial non-pharmacological intervention in the treatment of hypertension, we believe it to be important to uncover any sex differences in the effects of exercise, as this could provide valuable information allowing practitioners to develop more efficient treatment plans for both men and women with hypertension.

\section{Acknowledgements}

This study was partially funded by a research grant from the Appalachian State University Research Council. (Scott Collier, PI). The publication is funded in part by the Society for Women's Health Research (SWHR) and the Department of Health Leisure and Exercise Science at Appalachian State University.

\section{Author details}

${ }^{1}$ Vascular Biology and Autonomic Studies Laboratory, Appalachian State University, 111 Rivers Street, Boone, NC 28608, USA. ${ }^{2}$ Department of Medicine, SUNY Upstate Medical University 750 East Adams Street, Syracuse, NY 13210, USA. ${ }^{3}$ Department of Kinesiology and Community Health, University of Illinois at Urbana-Champaign, 906 South Goodwin Ave, Urbana IL 61801, USA. ${ }^{4}$ Center for the Study of Sex Differences in Health, Aging and Disease, Georgetown University, 4000 Reservoir Road, NW, Washington, DC 20057, USA.

\section{Authors' contributions}

SC conceived of the study, collected data and wrote the manuscript; VF recruited subjects, aided in study conception and provided clinical insight for the paper; KS helped in the study conception, ran all of the assays, edited the paper and provided clinical knowledge; PS helped in data collection and writing of the manuscript; $\mathrm{HJ}$ ran all of the assays and helped with the Methods section of the paper; HS aided in study conception and 
provided clinical insight for the paper; and BF helped with the study conception and manuscript editing. All authors read and approved the final manuscript.

\section{Competing interests}

The authors declare that they have no competing interests.

Received: 6 April 2011 Accepted: 25 August 2011

Published: 25 August 2011

\section{References}

1. Safar ME, Smulyan H: Hypertension in women. Am J Hypertens 2004, 17:82-87.

2. Reckelhoff JF: Gender differences in the regulation of blood pressure. Hypertension 2001, 37:1199-1208.

3. Collier SR, Kanaley JA, Carhart R Jr, Frechette V, Tobin MM, Hall AK, Luckenbaugh AN, Fernhall B: Effect of 4 weeks of aerobic or resistance exercise training on arterial stiffness, blood flow and blood pressure in pre- and stage- 1 hypertensives. Journal of human hypertension 2008, 22:678-686.

4. Layne JE, Nelson ME: The effects of progressive resistance training on bone density: a review. Medicine and science in sports and exercise 1999, 31:25-30.

5. Winett RA, Carpinelli RN: Potential health-related benefits of resistance training. Prev Med 2001, 33:503-513.

6. Lohman T, Going S, Pamenter R, Hall M, Boyden T, Houtkooper L, Ritenbaugh C, Bare L, Hill A, Aickin M: Effects of resistance training on regional and total bone mineral density in premenopausal women: a randomized prospective study. J Bone Miner Res 1995, 10:1015-1024.

7. Phillips SA, Das E, Wang J, Pritchard K, Gutterman DD: Resistance and aerobic exercise protects against acute endothelial impairment induced by a single exposure to hypertension during exertion. J Appl Physiol 2011, 110:1013-1020

8. Pierce GL, Eskurza I, Walker AE, Fay TN, Seals DR: Sex-specific effects of habitual aerobic exercise on brachial artery flow-mediated dilation in middle-aged and older adults. Clin Sci (Lond) 2011, 120:13-23.

9. Ainsworth BE, Jacobs DR Jr, Leon AS: Validity and reliability of selfreported physical activity status: the Lipid Research Clinics questionnaire. Medicine and science in sports and exercise 1993, 25:92-98.

10. Katch F, Michael ED, Horvath SM: Estimation of body volume by underwater weighing: description of a simple method. J Appl Physiol 1967, 23:811-813.

11. Van Bortel LM, Duprez D, Starmans-Kool MJ, Safar ME, Giannattasio C, Cockcroft J, Kaiser DR, Thuillez C: Clinical applications of arterial stiffness, Task Force III: recommendations for user procedures. Am J Hypertens 2002, 15:445-452.

12. Higashi Y, Sasaki S, Kurisu S, Yoshimizu A, Sasaki N, Matsuura H, Kajiyama G, Oshima T: Regular aerobic exercise augments endothelium-dependent vascular relaxation in normotensive as well as hypertensive subjects: role of endothelium-derived nitric oxide. Circulation 1999, 100:1194-1202.

13. Hagberg JM, Montain SJ, Martin WH, Ehsani AA: Effect of exercise training in 60- to 69-year-old persons with essential hypertension. Am J Cardiol 1989, 64:348-353.

14. Harris KA, Holly RG: Physiological response to circuit weight training in borderline hypertensive subjects. Medicine and science in sports and exercise 1987, 19:246-252.

15. Harvey PJ, Morris BL, Kubo T, Picton PE, Su WS, Notarius CF, Floras JS: Hemodynamic after-effects of acute dynamic exercise in sedentary normotensive postmenopausal women. Journal of hypertension 2005 23:285-292.

16. Higashi Y, Sasaki S, Sasaki N, Nakagawa K, Ueda T, Yoshimizu A, Kurisu S, Matsuura H, Kajiyama G, Oshima T: Daily aerobic exercise improves reactive hyperemia in patients with essential hypertension. Hypertension 1999, 33:591-597.

17. Okamoto T, Masuhara M, Ikuta K: Home-based resistance training improves arterial stiffness in healthy premenopausal women. Eur J Appl Physiol 2009, 107:113-117.

18. Miyachi M, Kawano H, Sugawara J, Takahashi K, Hayashi K, Yamazaki K, Tabata I, Tanaka H: Unfavorable Effects of Resistance Training on Central Arterial Compliance: A Randomized Intervention Study. Circulation 2004 110:2858-2863.
19. Bertovic DA, Waddell TK, Gatzka CD, Cameron JD, Dart AM, Kingwell BA: Muscular strength training is associated with low arterial compliance and high pulse pressure. Hypertension 1999, 33:1385-1391.

20. Okamoto T, Masuhara M, Ikuta K: Upper but not lower limb resistance training increases arterial stiffness in humans. Eur J Appl Physiol 2009, 107:127-134.

21. Casey DP, Pierce GL, Howe KS, Mering MC, Braith RW: Effect of resistance training on arterial wave reflection and brachial artery reactivity in normotensive postmenopausal women. Eur J Appl Physiol 2007, 100:403-408

22. Yoshizawa M, Maeda S, Miyaki A, Misono M, Saito Y, Tanabe K, Kuno S, Ajisaka R: Effect of 12 weeks of moderate-intensity resistance training on arterial stiffness: a randomised controlled trial in women aged 32-59 years. Br J Sports Med 2009, 43:615-618.

23. Casey DP, Beck DT, Braith RW: Progressive resistance training without volume increases does not alter arterial stiffness and aortic wave reflection. Exp Biol Med (Maywood) 2007, 232:1228-1235.

24. Rakobowchuk M, McGowan CL, de Groot PC, Bruinsma D, Hartman JW, Phillips SM, MacDonald MJ: Effect of whole body resistance training on arterial compliance in young men. Exp Physiol 2005, 90:645-651.

25. Cortez-Cooper MY, DeVan AE, Anton MM, Farrar RP, Beckwith KA, Todd JS, Tanaka $\mathrm{H}$ : Effects of high intensity resistance training on arterial stiffness and wave reflection in women. Am J Hypertens 2005, 18:930-934.

26. Tanaka H, Dinenno FA, Monahan KD, Clevenger CM, DeSouza CA, Seals DR: Aging, habitual exercise, and dynamic arterial compliance. Circulation 2000, 102:1270-1275.

27. Moreau KL, Donato AJ, Seals DR, DeSouza CA, Tanaka H: Regular exercise, hormone replacement therapy and the age-related decline in carotid arterial compliance in healthy women. Cardiovasc Res 2003, 57:861-868.

28. Arbab-Zadeh A, Dijk E, Prasad A, Fu Q, Torres P, Zhang R, Thomas JD, Palmer D, Levine BD: Effect of aging and physical activity on left ventricular compliance. Circulation 2004, 110:1799-1805.

29. Ferrier KE, Waddell TK, Gatzka CD, Cameron JD, Dart AM, Kingwell BA: Aerobic exercise training does not modify large-artery compliance in isolated systolic hypertension. Hypertension 2001, 38:222-226.

30. Redfield MM, Jacobsen SJ, Borlaug BA, Rodeheffer RJ, Kass DA: Age- and gender-related ventricular-vascular stiffening: a community-based study. Circulation 2005, 112:2254-2262.

31. Fagard RH: Exercise is good for your blood pressure: effects of endurance training and resistance training. Clin Exp Pharmacol Physiol 2006, 33:853-856.

32. Kelley GA, Kelley KS: Progressive resistance exercise and resting blood pressure: A meta-analysis of randomized controlled trials. Hypertension 2000, 35:838-843.

33. Thijssen DH, Dawson EA, Tinken TM, Cable NT, Green DJ: Retrograde flow and shear rate acutely impair endothelial function in humans. Hypertension 2009, 53:986-992.

34. Green DJ: Exercise training as vascular medicine: direct impacts on the vasculature in humans. Exerc Sport Sci Rev 2009, 37:196-202.

35. Fukai T, Siegfried MR, Ushio-Fukai M, Cheng Y, Kojda G, Harrison DG: Regulation of the vascular extracellular superoxide dismutase by nitric oxide and exercise training. J Clin Invest 2000, 105:1631-1639.

36. Hambrecht R, Adams V, Erbs S, Linke A, Krankel N, Shu Y, Baither $Y$, Gielen S, Thiele H, Gummert JF, et al: Regular physical activity improves endothelial function in patients with coronary artery disease by increasing phosphorylation of endothelial nitric oxide synthase. Circulation 2003, 107:3152-3158.

37. Sessa WC, Pritchard K, Seyedi N, Wang J, Hintze TH: Chronic exercise in dogs increases coronary vascular nitric oxide production and endothelial cell nitric oxide synthase gene expression. Circ Res 1994, 74:349-353.

doi:10.1186/2042-6410-2-9

Cite this article as: Collier et al:: Sex differences in resting hemodynamics and arterial stiffness following 4 weeks of resistance versus aerobic exercise training in individuals with pre-hypertension to stage 1 hypertension. Biology of Sex Differences 2011 2:9. 